\title{
Transcranial stimulation and cognition
}

\author{
CARLO MINIUSSI ${ }^{1,2} *$ AND MANUELA RUZZOLI $^{3}$ \\ ${ }^{I}$ Department of Clinical and Experimental Sciences, National Institute of Neuroscience, \\ University of Brescia, Brescia, Italy \\ ${ }^{2}$ Cognitive Neuroscience Section, IRCCS Centro San Giovanni di Dio Fatebenefratelli, Brescia, Italy \\ ${ }^{3}$ Department de Tecnologies de la Informació i les Comunicacions, Universitat Pompeu Fabra, Barcelona, Spain
}

\section{INTRODUCTION}

A major breakthrough in understanding where and when cognitive functions occur in the brain, as well as the mechanisms that underlie perceptual and cognitive functions, was provided by the introduction of modern neuroimaging technology, including noninvasive brain stimulation (NIBS). NIBS methods aim to induce changes in the activity of a specific area of the brain, with the final effect of altering the performance of a behavioral task performed by that area. NIBS techniques include transcranial magnetic stimulation (TMS) and transcranial electrical stimulation (tES) (see Chapter 27).

Both TMS and tES can transiently influence behavior by altering neuronal activity, which may have facilitatory or inhibitory behavioral effects. The relevance of NIBS in recent years as a tool in cognitive neuroscience stems mainly from its ability transiently to affect the function of the targeted cortical area, thereby altering behavior via a causal approach. This approach has been called a virtual lesion (Walsh and Cowey, 1998; Pascual-Leone et al., 1999) or, more recently, a perturbation. Therefore, NIBS can establish the functional participation of a brain area in an ongoing specific cognitive process based on where the stimulus is delivered, as well as the timing of its activity based on when the stimulus is delivered. NIBS has the advantage of combining lesion and neuroimaging approaches, thereby allowing more information about functionally relevant areas to be obtained (Walsh and Cowey, 1998). The opportunity to evaluate the mechanisms of brain function has introduced intriguing possibilities in basic cognitive neuroscience (Sandrini et al., 2011) and cognitive rehabilitation to promote cognitive plasticity in several pathological conditions (Miniussi et al., 2008) (see Table 56.1).

With TMS (see Chapter 27), a strong transient magnetic field, a defined pulse, is delivered through a coil to induce a transitory electric current at the cortical surface beneath the coil. The pulse causes depolarization of the cell membranes (Barker et al., 1985, 1987) and transsynaptic depolarization or hyperpolarization of cortical neuronal populations. TMS can be applied as one stimulus at a time (single-pulse TMS, spTMS), pairs of stimuli separated by a variable and programmable interval (paired-pulse TMS), or patterned stimulation (for a precise classification see Rossi et al., 2009). When multiple TMS stimuli are delivered in trains, conventional and patterned protocols of repetitive stimulation can be used. For conventional protocols, the term repetitive TMS (rTMS) is used universally. Patterned rTMS refers to a repetitive application of short rTMS bursts with a high inner frequency that is separated by short pauses with no stimulation. To date, theta-burst stimulation (TBS) has been the most commonly used method of patterned rTMS (Huang et al., 2005). Recently, quadripulse stimulation (QPS) has been become a patterned rTMS procedure (Hamada et al., 2008).

For the tES technique (see Chapter 27), the stimulation involves the application of weak electric currents directly to the scalp through a pair of electrodes. These currents generate an electrical field over the brain that modulates the neuronal activity according to the modality of the application, which can be direct (anodal or cathodal transcranial direct current stimulation, tDCS),

*Correspondence to:Carlo Miniussi, Department of Clinical and Experimental Sciences, Neuroscience section,University of Brescia, Viale Europa 11, 25123 Brescia, Italy. Tel: (+39) 0303717 441, E-mail: carlo.miniussi@cognitiveneuroscience.it 
Table 56.1

Summary of the noninvasive brain stimulation techniques mentioned in the text and their main features

\begin{tabular}{|c|c|c|c|c|}
\hline NIBS technique & Advantages & Disadvantages & Application & Relevant references \\
\hline spTMS & $\begin{array}{l}\text { Good spatial and } \\
\text { excellent temporal } \\
\text { resolution. Direct } \\
\text { evaluation of motor } \\
\text { and visual cortical } \\
\text { excitability }\end{array}$ & $\begin{array}{l}\text { High cost. Limitations } \\
\text { to stimulation of } \\
\text { superficial areas of } \\
\text { the brain }\end{array}$ & $\begin{array}{l}\text { All areas of cognition. } \\
\text { Neurophysiological } \\
\text { evaluation of } \\
\text { motor corticospinal } \\
\text { pathways. Mental } \\
\text { chronometry }\end{array}$ & $\begin{array}{l}\text { Rossi et al., 2009; } \\
\quad \text { Sandrini et al., } 2011\end{array}$ \\
\hline Online rTMS & $\begin{array}{l}\text { Good spatial and } \\
\text { temporal resolution. } \\
\text { It covers a larger time } \\
\text { window (versus } \\
\text { spTMS), allowing } \\
\text { evaluation of the } \\
\text { online involvement of } \\
\text { the stimulated area. It } \\
\text { modulates neuronal } \\
\text { activity during the } \\
\text { execution of a specific } \\
\text { task. Short- and long- } \\
\text { term neuromodulatory } \\
\text { effects }\end{array}$ & $\begin{array}{l}\text { High cost. Limitations } \\
\text { to stimulation of } \\
\text { superficial areas of } \\
\text { the brain. Possible } \\
\text { undesired short- } \\
\text { and long-term } \\
\text { neuromodulatory } \\
\text { effects. Noisy. Can } \\
\text { induce involuntary } \\
\text { muscle movements. } \\
\text { No good sham } \\
\text { condition. Safety } \\
\text { concerns }\end{array}$ & $\begin{array}{l}\text { All areas of cognition. } \\
\text { Working on signal } \\
\text { versus noise } \\
\text { modulation. } \\
\text { Working under the } \\
\text { state-dependency } \\
\text { hypothesis. } \\
\text { Working under the } \\
\text { entrainment } \\
\text { hypothesis }\end{array}$ & \\
\hline tDCS & $\begin{array}{l}\text { Painless. Reliable sham } \\
\text { condition with no scalp } \\
\text { sensations. Low risk of } \\
\text { adverse effects and } \\
\text { seizure induction. } \\
\text { Modulates neuronal } \\
\text { activity during } \\
\text { execution of a specific } \\
\text { task. Low cost }\end{array}$ & $\begin{array}{l}\text { Poor spatial and } \\
\text { temporal resolution. } \\
\text { Stimulation of large } \\
\text { part of the brain. } \\
\text { Concurrent } \\
\text { modulation of area } \\
\text { under reference } \\
\text { electrode }\end{array}$ & $\begin{array}{l}\text { All areas of cognition. } \\
\text { In association with } \\
\text { cognitive training } \\
\text { (rehabilitation) }\end{array}$ & $\begin{array}{l}\text { Nitsche et al., 2008; } \\
\text { Nitsche and } \\
\text { Paulus, } 2011\end{array}$ \\
\hline tACS & $\begin{array}{l}\text { Does not have the } \\
\text { polarity constraints of } \\
\text { tDCS. Reliable sham } \\
\text { condition with no scalp } \\
\text { sensations. Can } \\
\text { stimulate at a specific } \\
\text { frequency. Low risk of } \\
\text { adverse effects and } \\
\text { seizure induction. } \\
\text { Low cost }\end{array}$ & $\begin{array}{l}\text { Poor spatial and } \\
\text { temporal resolution. } \\
\text { Stimulation of large } \\
\text { part of the brain. At } \\
\text { certain frequencies } \\
\text { induction of (retinal) } \\
\text { phosphenes that can } \\
\text { interfere with task } \\
\text { execution }\end{array}$ & & $\begin{array}{l}\text { Zaghi et al., 2010; } \\
\text { Paulus, } 2011\end{array}$ \\
\hline tRNS & $\begin{array}{l}\text { Does not have the } \\
\text { polarity constraints of } \\
\text { tDCS. Reliable sham } \\
\text { condition with no scalp } \\
\text { sensations. Low risk of } \\
\text { adverse effects and } \\
\text { seizure induction. } \\
\text { Low cost }\end{array}$ & $\begin{array}{l}\text { Poor spatial and } \\
\text { temporal resolution. } \\
\text { Stimulation of large } \\
\text { part of the brain. } \\
\text { Unclear physiological } \\
\text { effects }\end{array}$ & & $\begin{array}{l}\text { Terney et al., 2008; } \\
\text { Fertonani et al., } \\
2011\end{array}$ \\
\hline
\end{tabular}

This table is not exhaustive; refer to main text for further details.

rTMS, repetitive transcranial magnetic stimulation; spTMS, single-pulse transcranial magnetic stimulation; tACS, transcranial alternating current stimulation; tDCS, transcranial direct current stimulation; tRNS, transcranial random noise stimulation. 
alternating (transcranial alternating current stimulation, tACS), or random noise (transcranial random noise stimulation, tRNS). It has been shown that during tDCS the neurons respond by altering their firing rates (i.e., cathodal stimulation reduces spontaneous neuronal firing rates), whereas the anodal tDCS exerts the opposite effect (Creutzfeldt et al., 1962; Bindman et al., 1964; Nitsche and Paulus, 2000) (see Table 56.1).

Therefore, NIBS techniques affect neuronal excitation through different mechanisms. TMS induces a current that elicits action potentials in neurons i.e., neurostimulation (Ruohonen, 2003). In contrast, tES (i.e., tDCS) induces polarization that is too weak to elicit action potentials in cortical neurons i.e., neuromodulation. However, tES effectively modifies the evoked cortical response to afferent stimulation, as well as the postsynaptic activity of cortical neurons, by inducing a shift in the intrinsic neuronal excitability, as demonstrated by tDCS studies in animals (Bindman et al., 1962, 1964; Purpura and McMurtry, 1965; Bindman et al., 1979). Despite this difference, both techniques induce effects at the cerebral level that are comparable in many respects, as supported by empirical evidence. For example, changes induced by a single application of rTMS or tDCS are reversible, last from a few seconds to more than 1 hour, and are dependent upon similar neurophysiological mechanisms (Ziemann et al., 1998a, b; Liebetanz et al., 2002; Nitsche et al., 2003; Fitzgerald et al., 2005; Ridding and Ziemann, 2010).

In general, in the cognitive domain the final effects induced by NIBS depend on the technical parameters used during stimulation (e.g., intensity of stimulation, coil orientation, site of reference electrode, current flow direction, focality and depth of stimulation, duration and frequency of stimulation, and time of application), as well as the possible interactions between these factors (for a recent review on the methodological aspects of NIBS in cognitive neuroscience see Sandrini et al., 2011). The effects also depend on several variables related to the stimulated subject, including age, eventual treatments, and the cognitive state of the subject (Silvanto et al., 2008b; Landi and Rossini, 2009; Miniussi et al., 2010; Nitsche and Paulus, 2011). This basic information and the selection of opportune parameters are essential for planning NIBS studies. Nevertheless, the mechanisms of action of NIBS as they relate to cognition remain unclear. Moreover, in the context of cognitive neuroscience, a theoretical framework is also needed to interpret the NIBS-induced behavioral effects beyond what has been possible with the simple perturbation approaches utilized thus far.

This chapter aims to present possible frameworks for a NIBS approach that could provide more information about the mechanisms of human brain function beyond what can be gained from the perturbation-localization method alone.

\section{TRANSCRANIAL MAGNETIC STIMULATION}

Understanding the effects of TMS in relation to cognition remains a challenge. The main idea, which has persisted over different theoretical frameworks for 25 years, is that if area $\mathrm{X}$ is involved in cognitive process $\mathrm{Y}$, the perturbation of the activity of area $\mathrm{X}$ will result in altered performance in process Y. In this sense, TMS overcomes the fundamental limits of the correlative approaches of other imaging techniques, such as functional magnetic resonance imaging (fMRI), positron emission tomography (PET), and electroencephalography (EEG), and provides an opportunity to test causal relationships between brain and cognition noninvasively.

The first step in reading cognition from TMS is to establish the role of different TMS parameters (e.g., intensity, protocol type, frequency) on behavioral and physiological outcomes. As an example, for the motor cortex, low frequencies $(\leq 1 \mathrm{~Hz})$ in a TMS protocol lead to a reduction in cortical excitability, whereas high frequencies $(\geq 5 \mathrm{~Hz})$ produce an opposite pattern of results (Fitzgerald et al., 2006; Rothkegel et al., 2010). This physiological evidence was directly translated to cognition based on the assumption that the suppression of cortical excitability impairs performance; however, this statement lacks adequate experimental support (see Sandrini et al., 2011, p. 521).

\section{Virtual lesion hypothesis}

The first hypothesis concerning TMS application was that of the "virtual lesion" (Walsh and Cowey, 1998; PascualLeone et al., 1999). Under this framework, TMS made it possible to study the functional role of a target brain area without any confounding factors typical of lesions in patients (e.g., compensation mechanisms, diaschisis, and dimension of the lesion). Similarly, TMS was considered to be able to induce transitory and reversible lesions in normal subjects. Since its initial application in cognitive neuroscience (Amassian et al., 1989), TMS has been used successfully to test the putative roles of many areas in regard to different types of cognitive task, such as visual awareness, language, crossmodal perception, memory, attention, and motor or mathematical cognition (for a review see other chapters in this section). The term localization approach usually refers to this approach, and implies the possibility of ascertaining where cognition occurs in the brain. In this sense, TMS borrowed experimental hypotheses from neuropsychology (patients with lesions) and, after extensive testing, proved most of them. Therefore, TMS and the virtual lesion hypothesis offered the possibility to produce final, ultimate confirmations for many previous hypotheses. 
TMS has also been employed to study when a cognitive event occurs. Mental chronometry has been applied to perceptual (Seyal et al., 1992; Corthout et al., 1999a, b) or higher-order cognitive processes (Ashbridge et al., 1997; Mottaghy et al., 2003; Kahn et al., 2005; Harris et al., 2008a; Ruzzoli et al., 2011b). In this sense, spTMS is more appropriate because it exploits the high temporal resolution of the machine, which is on the order of milliseconds. Mental chronometry has been useful in defining the temporal activation of singular brain areas and also in ascertaining the relative roles of different areas in the same cognitive process. For example, Laycock et al. (2007) reviewed the critical time windows for the inhibition of motion processing for visual cortices i.e., V1 and V5/Mt stimulation. The latter showed a double time window of activation, i.e., a late period between 100 and $150 \mathrm{~ms}$ and an early activation prior to the onset of motion (from-30 to $30 \mathrm{~ms}$ ). The authors proposed two hypotheses to explain the controversial early activation: a direct antidromic TMS interference with the lateral geniculate nucleus of thalamus or, alternatively, a disruption of the attention mechanism of expectation. Finally, V1 has been found to play a fundamental role in motion processing between 90 and $200 \mathrm{~ms}$ (Laycock et al., 2007; see also Stevens et al., 2009).

\section{Signal versus noise}

Although it is simple and direct, the virtual lesion hypothesis is only metaphor because the functional mechanism of TMS remains unclear (Miniussi et al., 2010). The virtual lesion hypothesis addresses the impairment of performance (concerning behavioral data); however, existing evidence does not support this aspect of the hypothesis (e.g., Topper et al., 1998; Cappa et al., 2002; Grosbras and Paus, 2003). Furthermore, it is not clear how the "virtual lesion" is generated. It is possible that TMS suppresses the relevant signal or induces (random) neural noise in the stimulated area; both mechanisms would impair performance but would do so through completely different mechanisms of action (Miniussi et al., 2010; Ruzzoli et al., 2010). Following this reasoning, Harris et al. (2008b) applied spTMS to the primary visual cortex during a Gabor orientation discrimination task. Consistent with the psychophysical model of their hypotheses (threshold versus noise function), they found that TMS significantly decreased the strength of the visual signal. In contrast, Ruzzoli et al. (2010) applied rTMS $(15 \mathrm{~Hz})$ to the V5/Mt during a motion direction discrimination task using a random-dot kinematogram; they found that TMS increased the neural noise. However, when spTMS was employed in the same cortical area (V5/Mt) during the same task (motion direction discrimination), the results indicated a signal reduction
(Ruzzoli et al., 2011a). It is important to note that the paradigms adopted in the studies cited above are distinct (spTMS versus rTMS); this difference may explain the opposing results. Indeed, whereas spTMS might affect a limited population of neurons, it is likely that rTMS activates a larger population, most of which are irrelevant for the execution of the task through temporal summation effects, and that the resulting noise is generated from a nonlinear interaction with the ongoing neural activity (Ruzzoli et al., 2011a). Thus, from a physiological standpoint, the effects of a series of pulses might be different from the effect of a single pulse. Repetitive stimulation is characterized by a train of pulses discharged in a brief time whose length is dictated by frequency; therefore, rTMS might influence the neural synchronization within and between cortical areas involved in the execution of the task (Allen et al., 2007; Pasley et al., 2009).

Forward progress from the virtual lesion metaphor to the definition of the precise mechanisms of action (signal suppression or noise generation) made it possible to test new hypotheses and to refresh the prospective applications of TMS. TMS essentially introduces activity by depolarizing neurons (Ruohonen, 2003); therefore, its signal suppression and induction of neural noise are counterintuitive. Physiologically, TMS might decrease the signal strength by altering TMS-induced membrane permeability. Alternatively, would be possible to achieve the same result if TMS enhances inhibitory $\gamma$-aminobutyric acid (GABA)ergic activity or reduces facilitatory glutamatergic activity (Moliadze et al., 2003; Mantovani et al., 2006; Yue et al., 2009). TMS may also induce a temporary saturation of neuronal function that leads to a loss of the information encoded by the activity of the affected neurons. In contrast, the effects of TMS can be considered to represent the introduction of neural noise, as TMS-induced neural activity is induced artificially and does not occur naturally in the system. Thus, TMS could modify the activity of a system by altering the information carried by a precise induced neural firing pattern. This effect could be interpreted as the generation of neural activity (noise) by TMS with respect to the relevant information carried by the stimulated area (signal).

One of the limits of the virtual lesion hypothesis is that it postulates only the impairment of performance; any positive results were addressed as a "paradoxical effect." With the neural noise generation hypothesis, it is easy to explain either output because noise can be considered to be the major source of variability, which results in the impairment of performance. However, in nonlinear systems, information can be better processed within an optimal level of noise, as suggested by the concept of stochastic resonance (Moss et al., 2004; Miniussi 
et al., 2010; Ruzzoli et al., 2010; Schwarzkopf et al., 2011), wherein the induced noisy activity might be synchronized with the ongoing relevant signal (Stein et al., 2005; Ermentrout et al., 2008). It is worth noting that experimental evidence supporting the noise generation hypothesis has been provided only by rTMS studies (Ruzzoli et al., 2010; Schwarzkopf et al., 2011).

\section{Pedestal effect}

An alternative explanation for both negative and positive results in TMS studies is provided by psychophysiology (Crowder et al., 2006; Solomon, 2009) and the so-called pedestal effect. Indeed, the dipper function (which plots signal strength against the just noticeable difference. JND) is characterized by an initial (at weak stimulus intensity) "dip" where the JND decreases as the stimulus strength increases (indicating better performance). As described by Weber's law, when a strong input occurs the JND increases as the stimulus strength increases. This alternative hypothesis has been directly tested in visual perception (Abrahamyan et al., 2011), where spTMS (note that a single-pulse paradigm was utilized here) was applied at different intensities over V1 to measure concurrently the threshold for plaid detection in a two-interval forced-choice study. It was found that, at intensities below the phosphene threshold (weak signal), TMS significantly improved the performance in comparison with the control condition, but only for contralateral stimuli. The study also confirmed a well known effect in vision studies: higher TMS intensity (above the phosphene threshold) increases visual threshold (Abrahamyan et al., 2011). Therefore, TMS would act as a "pedestal" when applied at a low intensity and primarily affect the signal strength as a function of stimulation intensity, with high intensity strengthening the signal and low intensity reducing it. In apparent contrast, stochastic resonance and the pedestal effect might share the same mechanism, as suggested by the finding that the pedestal effect is reduced in the presence of weak noise (Goris et al., 2008).

\section{State dependency}

As described above, we cannot deduce pure TMSinduced effects, because the target brain area and its functional state must always be kept in consideration. The state dependency argument was first explicitly articulated in the TMS field by Silvanto and Muggleton (2008) (see also Sack and Linden, 2003; Silvanto et al., 2008b), but its origins are well known (Sherrington, 1910). Importantly, the effects of TMS are proportional to the level of neuronal activation during the application of the stimulus (Epstein and Rothwell, 2003). In the motor system, the amplitude of the motor evoked potential can be increased by the voluntary contraction of the target muscle (Rothwell et al., 1987). According to state dependency, TMS will affect the less active neurons within the stimulated area. Silvanto et al. (2007) adapted subjects to a red/green screen. After 30 seconds, the delivery of a spTMS over the occipital cortex elicited phosphenes that took on the same color of the adapting stimulus. Similarly, adaptation to a motion translation direction allowed TMS to facilitate the detection of motion in the adapted direction while impairing the detection of motion in the opposite direction (Silvanto et al., 2008a). State dependency has been tested and validated under different experimental protocols (i.e., priming or adaptation) and for different brain areas (Cattaneo et al., 2008, 2009, 2010).

Despite the amount of data that converge to support state dependency, we prefer not to label state dependency as a hypothesis because it does not provide an explicit mechanism of how TMS affects cognition. However, we do consider that the functional state of a given area (mostly determined by the task manipulation) is extremely important in interpreting the TMS data (and is too frequently ignored in the TMS literature, as suggested by Silvanto et al., 2008b), because it allows us to discriminate between neural populations within a single cerebral area based on their functionality. These findings suggest that the effects are sensitive to changes in the cortical state, which raises the intriguing possibility that the administration of TMS (and also tES) while a subject performs a behavioral task may permit specific circuitry to be targeted.

\section{Entrainment}

The final and more recent application of TMS is known as the entrainment hypothesis (Thut and Miniussi, 2009). Oscillation frequencies are often linked to different cognitive statuses (Varela et al., 2001; Buzsaki and Draguhn, 2004; Canolty and Knight, 2010). For example, the alpha frequency $(8-14 \mathrm{~Hz})$ is associated with visual attention, orientation, and perception (Worden et al., 2000; Thut et al., 2006). The hypothesis is that a rhythmic external force (TMS) can perturb the frequency of the system. Therefore, according to the entrainment hypothesis, rhythmic brain stimulation (i.e., a repetitive paradigm) might induce the same rhythm in the brain.

This hypothesis was derived from evidence that alpha-band oscillation at the occipital-parietal location increases and decreases ipsilateral and contralateral to the visual attended side, respectively (Sauseng et al., 2005; Thut et al., 2006; Foxe and Snyder, 2011). Furthermore, alpha power has been found to be inversely correlated with perceptual outcome, which means that a greater alpha-band activity prior to target presentation 
results in worse visual detection (Thut et al., 2006). However, whether alpha power modulation is just an epiphenomenon or actually has functional relevance is a matter of debate. Romei et al. (2010) aimed to evaluate the alpha power modulation using TMS; they applied rhythmic TMS $(10,20$, and $5 \mathrm{~Hz})$ to the parietal-occipital cortex just before a peripheral visual target presentation. They asked the participant to press a button only when the stimulus could be perceived. They found that $10-\mathrm{Hz}$ (alpha-band) rTMS selectively impaired the performance for stimuli applied contralaterally to TMS and improved the performance for stimuli presented ipsilaterally; the effect was specific for $10-\mathrm{Hz}$ TMS (versus 20 or $5 \mathrm{~Hz}$ ). This result strongly suggests a functional role for the alpha frequency in visual attentional bias and the possibility to induce a specific frequency by externally employing brain stimulation (Romei et al., 2010). More recently, Thut et al. (2011b) directly tested the entrainment hypothesis by means of a concurrent EEG-TMS experiment, where they first determined the source of the parietal-occipital attention to be alpha modulation and calculated the individual alpha frequency (magnetoencephalography (MEG) study), after which they applied rTMS at the individual alpha power while recording the EEG activity at rest. The results confirmed the three predictions of the entrainment hypothesis: the induction of a specific frequency after TMS, the enhancement of oscillation during TMS stimulation because of synchronization, and a phase alignment of the induced frequency and the ongoing activity (Thut et al., 2011b).

Because of the entrainment hypothesis, the theoretical framework regarding interpretation of TMS data has changed again. The hypothesis makes clear predictions, mainly regarding repetitive TMS paradigms (frequency engagement), but also argues the possibility of inducing phase resetting via spTMS (Thut et al., 2011a; Veniero et al., 2011). This new hypothesis completely overcomes the localization approach and is conducive to gaining knowledge about how the brain works rather than where a single process takes place. In this sense, the TMS is likely the best available method to test a renewed topic in neuroscience: the role of brain oscillations.

\section{Brain networks}

In conjunction with other imaging techniques (fMRI, EEG, PET, MEG), TMS has also been utilized to test brain networks, i.e., the functional effects of a given area on distal locations during a specific task. This multimodal imaging approach (Siebner et al., 2009; Miniussi and Thut, 2010) permits the assessment of the local impact of TMS on neural processing by means of objective measures of cortical reactivity (over the directly targeted area). Moreover, it provides an assessment of the remote effects of TMS (on neural processing in distal brain regions). Importantly, local activation by a magnetic pulse is expected to spread to anatomically or functionally connected areas that can be simultaneously traced by fMRI, PET, or multichannel EEG recording. Because the brain operates through flexible and interactive distributed networks, we expect that the modification of a node of the network affects the entire network. If brain stimulation is applied when the system is in a given functional state, it will expose the corticocortical network that is interconnected with the target area at time of TMS and, eventually, how that network can be modified by a specific state (Massimini et al., 2005).

However, understanding how different brain areas are interconnected does not tell us how those areas interact to sustain perception or cognition. Recently, TMS-EEG coregistration has been used to test connectivity. Morishima et al. (2009) hypothesized that the stimulation of the prefrontal areas of the attentional network would induce the spread of current toward the anatomically connected posterior regions, and that the direction and amount of the current spread could be modulated depending on the functional status of the neural network, which is determined by the task being performed by the subjects. During cued attention to visual features, TMS of the frontal eye field induced activity in different posterior visual areas depending on the specific nature of the feature (Morishima et al., 2009). Moreover, the TMSEEG coregistration approach used by Morishima et al. (2009) provided additional information about the interplay between the prefrontal and posterior areas. The TMS effects occurred between 20 and $40 \mathrm{~ms}$ after the pulse, which suggests that they were not the result of rerouting via other areas but that there was a direct corticocortical signal transmission from frontal to posterior regions. The main strength of this approach is the ability to perform real-time measurements of whole-brain activity changes while subjects are performing a task during which specific areas of the network are stimulated concurrently, which yields functional segregation from the surrounding areas.

\section{TRANSCRANIAL ELECTRICAL STIMULATION}

As previously reported, tES does not induce action potentials but rather modulates neuronal response thresholds. This change in the neuronal threshold results from a change in membrane permeability (Liebetanz et al., 2002) that influences the response of the taskrelated network. Therefore, the network is modulated by tES and by the task carried by the subject. It is important to understand the mechanism underlying this type of stimulation, which, in contrast to TMS, is a constant 
stimulation. Constant stimulation can induce neurophysiological homeostasis, which serves to maintain neural activity within a normal functional range (Siebner et al., 2004). This type of mechanism could alter the final effect of the stimulation in terms of excitatory or inhibitory responses of the stimulated area. Therefore, it is not necessarily true that anodal stimulation indicates behavioral facilitation and cathodal stimulation indicates behavioral inhibition, although the effects should once again be considered in the context of a complex framework.

\section{Transcranial direct current stimulation}

It has been shown that the effects of tDCS are induced by membrane depolarization (anodal) and hyperpolarization (cathodal) (Liebetanz et al., 2003). Therefore, tDCS may "prime" the system by increasing or decreasing the excitability of the system, or by decreasing or increasing the threshold response. However, these observations are based mainly on the motor system (Nitsche et al., 2008), and the effects on behavior are often not a clearcut with anodal stimulation inducing facilitation, and cathodal stimulation inducing inhibition (for a review see Nitsche et al., 2008; Nitsche and Paulus, 2011).

The use of $\mathrm{tDCS}$ in cognitive neuroscience is still considered to be in its infancy. From a methodological perspective, most general concerns for TMS are valid for tDCS, with some exceptions. tDCS does not induce depolarization and therefore will not induce the firing of neurons that are not near threshold; this means that neurons not engaged in the task are less likely to discharge. Therefore, tDCS-induced effects are more likely to be sensitive to the state of the network or to that of the stimulated area, which suggests that, if tDCS is applied during task execution, a specific circuitry can be targeted (e.g., the circuitry modulated by the task or taskrelated network). The spatial and temporal resolution of the tDCS effects are reduced compared with TMS, but this drawback may be overcome by the application discussed above; the threshold of activation or deactivation for a task-related network will be modulated than that of other networks by tDCS unless the signal in that network is strong enough to produce a univocal response. Therefore, tES would act as a "pedestal" for the network signal strength when stimulation is applied anodally, thereby strengthening it. Alternatively, tES would reduce the signal strength when stimulation is applied cathodally. Facilitative behavioral effects have been highlighted in regard to working memory (Fregni et al., 2005; Ohn et al., 2008), pitch memory (Vines et al., 2006), perception (Antal et al., 2004), and language (Iyer et al., 2005; Sparing et al., 2008). For example, Fertonani et al. (2010) explored the effects of tDCS on a picture-naming language task and found that anodal tDCS applied to the left dorsolateral prefrontal cortex improved naming performance, which suggests that anodal tDCS over the prefrontal cortex increased the signal intensity.

\section{Transcranial random noise stimulation}

tRNS involves the application of a random electrical oscillation spectrum over the cortex. tRNS can be applied in three frequency ranges: the entire spectrum from 0.1 to $640 \mathrm{~Hz}$, in the low band $(0.1-100 \mathrm{~Hz})$, or in the high band $(101-640 \mathrm{~Hz})$. This technique is newer than other tES applications; therefore, speculation about its possible mechanisms of action in cognition is rare to date.

Terney et al. (2008) recently showed that 10 minutes of tRNS on the motor cortex at high frequency is able positively to modulate cortical excitability (i.e., increase the amplitude of motor evoked potentials) in a manner that persists after the conclusion of stimulation. Behavioral improvement in a motor learning task also resulted from the application of the entire frequency spectrum (Terney et al., 2008). In a recent study, Fertonani et al. (2011) applied tRNS to the visual system and compared the high-/low-frequency bands with other tES techniques (anodal/cathodal tDCS). High-frequency tRNS on the visual cortex of healthy subjects during a visual perceptual learning task was found to improve performance significantly more than anodal tDCS, which was previously thought to be the best method to modulate behavior positively. The authors suggested that the mechanism of action of tRNS might be based on the repeated subthreshold stimulations that prevent homeostasis of the system (Fertonani et al., 2011). This effect might potentiate the activity of the neural populations involved in a task and in turn facilitate brain plasticity by strengthening the synaptic transmission between neurons. Because tRNS is a repetitive, random, and subthreshold stimulation, it has been hypothesized that tRNS will induce the direct temporal summation of neural activity because the time constant of a neuron is sufficiently long to permit the summation of two stimuli presented in close succession (Fertonani et al., 2011). Thus, the mechanisms of action of tRNS might be based on repeated subthreshold stimulations that collectively prevent the homeostasis of the system (Fertonani et al., 2011).

The effects of tRNS may also be explained in the context of the stochastic resonance phenomenon (e.g., Miniussi et al., 2010); tRNS is, by definition, a stimulation that induces nonfinalized random activity in the system (i.e., noise). The presence of neuronal noise might enhance the sensitivity of the neurons to a given range of weak inputs (the neurons with the same directionality 
as the signal), thereby introducing a functionally useful noise to the signal and increasing the signal. Therefore, the effect of tRNS on neuronal activity may not just be the random addition of noise. Nevertheless, even if the term "random noise stimulation" can evoke such an explanation, no data currently allow us to draw a more concrete explanation within this framework.

\section{Transcranial alternating current stimulation}

A different hypothetical consideration should be drawn for tACS, even though it is still potentially considered within the "modulation" framework of tES. Electrical brain activity is characterized by oscillations, which are widely believed to play a functional role in various cognitive functions, such as perception, attention, memory, and motor behavior (Buzsaki and Draguhn, 2004; see also the section on Entrainment). In the last several decades, there has been a growing consensus that neuronal oscillations play a fundamental role in brain operation and that an understanding of neuronal oscillations is essential to understand brain functions (Schroeder and Lakatos, 2009). Like rTMS, a new theory concerning tACS effects suggests that brain stimulation techniques can modulate ongoing neuronal activity and related behavior by inducing specific brain oscillations (Schurmann et al., 2001). We can theoretically predict that this mechanism will produce a frequency "entrainment" in the stimulated cortical region or in the connected areas during a prolonged stimulation (Thut and Miniussi, 2009; Thut et al., 2011a, b). Using tACS (as for rTMS), it is possible to deliver an oscillatory current to the cortex in a frequency-specific manner to induce a particular oscillatory entrainment (Kanai et al., 2008).

Antal and colleagues (2008) were the first to apply tACS over the primary motor area with the objective of specifically influencing brain oscillations. Stimulation was applied at different frequencies $(1-45 \mathrm{~Hz})$ during an implicit motor learning task, and a significant improvement in performance was observed after only 7 minutes of $10-\mathrm{Hz}$ stimulation. Using tACS, Kanai et al. (2008) (see also Zaehle et al., 2010) reported that occipital stimulation most effectively induced phosphenes when applied at the alpha frequency in darkness; in contrast, the beta frequency was more effective in the light. In support of a causal role for sleep slow waves in memory consolidation, Marshall et al. (2006) observed that the induction of slow-wave frequencies during sleep enhances memory consolidation. In this respect, the so-called rhythmic approach (Thut and Miniussi, 2009; Miniussi et al., 2012) refers to the possibility of investigating how tACS interacts with oscillatory brain activity. Therefore, the confirmation that tACS can entrain cortical oscillations will demonstrate a causal relationship between brain oscillations and cognition (Thut and Miniussi, 2009).

Thus, we can assume that the effects of tES may potentiate the activity of neural populations involved in tasks that facilitate brain plasticity by strengthening synaptic communication/transmissions between neurons. Specifically, the modulation of the efficacy of synaptic transmission can alter excitability and activity in definite cortical networks that are specifically activated by the execution of the task; these changes correspond to an adjustment of the threshold response that correlates with cognitive plasticity at the behavioral level.

\section{FUTURE DIRECTIONS: COGNITIVE NEUROREHABILITATION AND NONINVASIVE BRAIN STIMULATION}

The use of NIBS to study and treat dysfunctions in psychiatric, stroke, and neurodegenerative patients has received much attention recently (see section above on therapeutic uses). In the cognitive domain, several studies (for a review see Miniussi et al., 2008) have shown improved behavioral performance in many cognitive tasks. For example, when rTMS was used in stimulation therapy, naming improvements were observed in patients with chronic vascular aphasia (for a review see Cotelli et al., 2011b), primary progressive aphasia (Finocchiaro et al., 2006), and Alzheimer's disease (Cotelli et al., 2011a). Increased attentional performance in patients with unilateral neglect associated with stroke was also found (for a review see Hesse et al., 2011).

Furthermore, tDCS has demonstrated the same potential as rTMS (see section above on therapeutic uses). Previous studies have shown that excitatory anodal tDCS in normal adults (Iyer et al., 2005; Floel et al., 2008; Sparing et al., 2008; Fertonani et al., 2010) and patients with aphasia (Baker et al., 2010; Cotelli et al., 2011b; Fiori et al., 2011; Fridriksson et al., 2011) enhances language performance. Recently, it has also been shown that a single tDCS session can ameliorate visuospatial attention deficits in stroke patients suffering from neglect (Sparing et al., 2009) and "partially restore" memory in patients with Alzheimer's disease (for a review see Boggio et al., 2011; Cotelli et al., 2012).

The mechanisms of action that we have described thus far were observed in healthy subjects but can be easily tested in patients. For example, possible mechanisms to support NIBS-induced cerebral plasticity have been proposed: both long-term potentiation and its opposite, long-term depression, have been postulated to underlie the effects of NIBS on cortical activity (Cooke and Bliss, 2006; Thickbroom, 2007; Ziemann and Siebner, 2008). In addition to these notions, information is available on the 
molecular and genetic aspects of NIBS-induced plasticity (Kleim et al., 2006; Zanardini et al., 2006; Zhang et al., 2007; Fritsch et al., 2010). Furthermore, functional and effective connectivity analysis can be employed to test dynamic brain reorganization in patients (Grefkes and Fink, 2011) and after NIBS application (Venkatakrishnan and Sandrini, 2012).

It is generally considered that the induction of changes in cortical excitability via brain stimulation leads to the reorganization of the functional network responsible for the impaired cognitive function. This reorganization might include the recruitment of compensatory networks, including contralateral homologous cortical regions, an additional recruitment of perilesional suboptimally functioning areas, or both. The reorganization might also represent an induced change in the balance of cortical-subcortical activity (Rushmore and Payne, 2003) or in the balance of activities in different neuroanatomical-behavioral networks (see Miniussi and Rossini, 2011).

Determining whether these NIBS techniques are useful for improving deficits in the cognitive domain will be a key goal of future research in the field of cognitive rehabilitation (Miniussi and Vallar, 2011).

The neural effects of NIBS protocols have the potential to offer important insights into the mechanisms that underlie the remarkable flexibility of the central nervous system, and they will help to test neurocognitive theories of the behavior-brain relationship. The opportunity to influence brain activity directly in a clear theoretical framework raises exciting possibilities for basic and clinical cognitive neuroscience research.

\section{REFERENCES}

Abrahamyan A, Clifford CW, Ruzzoli M et al. (2011). Accurate and rapid estimation of phosphene thresholds (REPT). PLoS One 6: e22342.

Allen EA, Pasley BN, Duong T et al. (2007). Transcranial magnetic stimulation elicits coupled neural and hemodynamic consequences. Science 317: 1918-1921.

Amassian VE, Cracco RQ, Maccabee PJ et al. (1989). Suppression of visual perception by magnetic coil stimulation of human occipital cortex. Electroencephalogr Clin Neurophysiol 74: 458-462.

Antal A, Nitsche MA, Kruse W et al. (2004). Direct current stimulation over V5 enhances visuomotor coordination by improving motion perception in humans. J Cogn Neurosci 16: 521-527.

Antal A, Boros K, Poreisz C et al. (2008). Comparatively weak after-effects of transcranial alternating current stimulation (tACS) on cortical excitability in humans. Brain Stimul 1: 97-105.

Ashbridge E, Walsh V, Cowey A (1997). Temporal aspects of visual search studied by transcranial magnetic stimulation. Neuropsychologia 35: 1121-1131.
Baker JM, Rorden C, Fridriksson J (2010). Using transcranial direct-current stimulation to treat stroke patients with aphasia. Stroke 41: 1229-1236.

Barker AT, Jalinous R, Freeston IL (1985). Non-invasive magnetic stimulation of human motor cortex. Lancet 1 : 1106-1107.

Barker AT, Freeston IL, Jalinous R et al. (1987). Magnetic stimulation of the human brain and peripheral nervous system: an introduction and the results of an initial clinical evaluation. Neurosurgery 20: 100-109.

Bindman LJ, Lippold OC, Redfearn JW (1962). Long-lasting changes in the level of the electrical activity of the cerebral cortex produced by polarizing currents. Nature 196: 584-585.

Bindman LJ, Lippold OC, Redfearn JW (1964). The action of brief polarizing currents on the cerebral cortex of the rat (1) during current flow and (2) in the production of long-lasting after-effects. J Physiol 172: 369-382.

Bindman LJ, Lippold OC, Milne AR (1979). Prolonged changes in excitability of pyramidal tract neurones in the cat: a post-synaptic mechanism. J Physiol 286: 457-477.

Boggio PS, Valasek CA, Campanha C et al. (2011). Noninvasive brain stimulation to assess and modulate neuroplasticity in Alzheimer's disease. Neuropsychol Rehabil 21: 703-716.

Buzsaki G, Draguhn A (2004). Neuronal oscillations in cortical networks. Science 304: 1926-1929.

Canolty RT, Knight RT (2010). The functional role of crossfrequency coupling. Trends Cogn Sci 14: 506-515.

Cappa SF, Sandrini M, Rossini PM et al. (2002). The role of the left frontal lobe in action naming: rTMS evidence. Neurology 59: 720-723.

Cattaneo Z, Rota F, Vecchi T et al. (2008). Using statedependency of transcranial magnetic stimulation (TMS) to investigate letter selectivity in the left posterior parietal cortex: a comparison of TMS-priming and TMS-adaptation paradigms. Eur J Neurosci 28: 1924-1929.

Cattaneo Z, Silvanto J, Pascual-Leone A et al. (2009). The role of the angular gyrus in the modulation of visuospatial attention by the mental number line. Neuroimage 44: 563-568.

Cattaneo L, Sandrini M, Schwarzbach J (2010). Statedependent TMS reveals a hierarchical representation of observed acts in the temporal, parietal, and premotor cortices. Cereb Cortex 20: 2252-2258.

Cooke SF, Bliss TV (2006). Plasticity in the human central nervous system. Brain 129: 1659-1673.

Corthout E, Uttl B, Walsh V et al. (1999a). Timing of activity in early visual cortex as revealed by transcranial magnetic stimulation. Neuroreport 10: 2631-2634.

Corthout E, Uttl B, Ziemann U et al. (1999b). Two periods of processing in the (circum)striate visual cortex as revealed by transcranial magnetic stimulation. Neuropsychologia 37: $137-145$.

Cotelli M, Calabria M, Manenti R et al. (2011a). Improved language performance in Alzheimer disease following brain stimulation. J Neurol Neurosurg Psychiatry 82: 794-797.

Cotelli M, Fertonani A, Miozzo A et al. (2011b). Anomia training and brain stimulation in chronic aphasia. Neuropsychol Rehabil 21: 717-741. 
Cotelli M, Manenti R, Miniussi C (2012). Nonpharmacological intervention for memory decline. Front Hum Neurosci 6: 46.

Creutzfeldt OD, Fromm GH, Kapp H (1962). Influence of transcortical d-c currents on cortical neuronal activity. Exp Neurol 5: 436-452.

Crowder NA, Price NS, Hietanen MA et al. (2006). Relationship between contrast adaptation and orientation tuning in V1 and V2 of cat visual cortex. J Neurophysiol 95: 271-283.

Epstein CM, Rothwell JC (2003). Therapeutic uses of rTMS. In: S Boniface, U Ziemann (Eds.), Plasticity in the Human Nervous System: Investigations with Transcranial Magnetic Stimulation. Cambridge University Press, Cambridge, pp. 246-263.

Ermentrout GB, Galan RF, Urban NN (2008). Reliability, synchrony and noise. Trends Neurosci 31: 428-434.

Fertonani A, Rosini S, Cotelli M et al. (2010). Naming facilitation induced by transcranial direct current stimulation. Behav Brain Res 208: 311-318.

Fertonani A, Pirulli C, Miniussi C (2011). Random noise stimulation improves neuroplasticity in perceptual learning. J Neurosci 31: 15416-15423.

Finocchiaro C, Maimone M, Brighina F et al. (2006). A case study of primary progressive aphasia: improvement on verbs after rTMS treatment. Neurocase 12: 317-321.

Fiori V, Coccia M, Marinelli CV et al. (2011). Transcranial direct current stimulation improves word retrieval in healthy and nonfluent aphasic subjects. J Cogn Neurosci 23: 2309-2323.

Fitzgerald PB, Benitez J, Oxley T et al. (2005). A study of the effects of lorazepam and dextromethorphan on the response to cortical $1 \mathrm{~Hz}$ repetitive transcranial magnetic stimulation. Neuroreport 16: 1525-1528.

Fitzgerald PB, Fountain S, Daskalakis ZJ (2006). A comprehensive review of the effects of rTMS on motor cortical excitability and inhibition. Clin Neurophysiol 117: 2584-2596.

Floel A, Rosser N, Michka O et al. (2008). Noninvasive brain stimulation improves language learning. J Cogn Neurosci 20: 1415-1422.

Foxe JJ, Snyder AC (2011). The role of alpha-band brain oscillations as a sensory suppression mechanism during selective attention. Front Psychol 2: 154.

Fregni F, Boggio PS, Nitsche M et al. (2005). Anodal transcranial direct current stimulation of prefrontal cortex enhances working memory. Exp Brain Res 166: 23-30.

Fridriksson J, Richardson JD, Baker JM et al. (2011). Transcranial direct current stimulation improves naming reaction time in fluent aphasia: a double-blind, shamcontrolled study. Stroke 42: 819-821.

Fritsch B, Reis J, Martinowich K et al. (2010). Direct current stimulation promotes BDNF-dependent synaptic plasticity: potential implications for motor learning. Neuron 66: 198-204.

Goris RL, Wagemans J, Wichmann FA (2008). Modelling contrast discrimination data suggest both the pedestal effect and stochastic resonance to be caused by the same mechanism. J Vis 8: 17.1-17.21.
Grefkes C, Fink GR (2011). Reorganization of cerebral networks after stroke: new insights from neuroimaging with connectivity approaches. Brain 134: 1264-1276.

Grosbras MH, Paus T (2003). Transcranial magnetic stimulation of the human frontal eye field facilitates visual awareness. Eur J Neurosci 18: 3121-3126.

Hamada M, Terao Y, Hanajima R et al. (2008). Bidirectional long-term motor cortical plasticity and metaplasticity induced by quadripulse transcranial magnetic stimulation. J Physiol 586: 3927-3947.

Harris IM, Benito CT, Ruzzoli M et al. (2008a). Effects of right parietal transcranial magnetic stimulation on object identification and orientation judgments. J Cogn Neurosci 20: 916-926.

Harris JA, Clifford CW, Miniussi C (2008b). The functional effect of transcranial magnetic stimulation: signal suppression or neural noise generation? J Cogn Neurosci 20: 734-740.

Hesse MD, Sparing R, Fink GR (2011). Ameliorating spatial neglect with non-invasive brain stimulation: from pathophysiological concepts to novel treatment strategies. Neuropsychol Rehabil 21: 676-702.

Huang YZ, Edwards MJ, Rounis E et al. (2005). Theta burst stimulation of the human motor cortex. Neuron 45: 201-206.

Iyer MB, Mattu U, Grafman J et al. (2005). Safety and cognitive effect of frontal DC brain polarization in healthy individuals. Neurology 64: 872-875.

Kahn I, Pascual-Leone A, Theoret H et al. (2005). Transient disruption of ventrolateral prefrontal cortex during verbal encoding affects subsequent memory performance. J Neurophysiol 94: 688-698.

Kanai R, Chaieb L, Antal A et al. (2008). Frequencydependent electrical stimulation of the visual cortex. Curr Biol 18: 1839-1843.

Kleim JA, Chan S, Pringle E et al. (2006). BDNF val66met polymorphism is associated with modified experiencedependent plasticity in human motor cortex. Nat Neurosci 9: 735-737.

Landi D, Rossini PM (2009). Cerebral restorative plasticity from normal ageing to brain diseases: a "never ending story". Restor Neurol Neurosci 28: 349-366.

Laycock R, Crewther DP, Fitzgerald PB et al. (2007). Evidence for fast signals and later processing in human V1/V2 and V5/MT +: A TMS study of motion perception. J Neurophysiol 98: 1253-1262.

Liebetanz D, Nitsche MA, Tergau F et al. (2002). Pharmacological approach to the mechanisms of transcranial DC-stimulation-induced after-effects of human motor cortex excitability. Brain 125: 2238-2247.

Liebetanz D, Nitsche MA, Paulus W (2003). Pharmacology of transcranial direct current stimulation: missing effect of riluzole. Suppl Clin Neurophysiol 56: 282-287.

Mantovani M, Van Velthoven V, Fuellgraf H et al. (2006). Neuronal electrical high frequency stimulation enhances GABA outflow from human neocortical slices. Neurochem Int 49: 347-350.

Marshall L, Helgadottir H, Molle M et al. (2006). Boosting slow oscillations during sleep potentiates memory. Nature 444: 610-613. 
Massimini M, Ferrarelli F, Huber R et al. (2005). Breakdown of cortical effective connectivity during sleep. Science 309: 2228-2232.

Miniussi C, Rossini PM (2011). Transcranial magnetic stimulation in cognitive rehabilitation. Neuropsychol Rehabil 21: 579-601.

Miniussi C, Thut G (2010). Combining TMS and EEG offers new prospects in cognitive neuroscience. Brain Topogr 22: 249-256.

Miniussi C, Vallar G (2011). Brain stimulation and behavioural cognitive rehabilitation: a new tool for neurorehabilitation? Neuropsychol Rehabil 21: 553-559.

Miniussi C, Cappa SF, Cohen LG et al. (2008). Efficacy of repetitive transcranial magnetic stimulation/transcranial direct current stimulation in cognitive neurorehabilitation. Brain Stimul 1: 326-336.

Miniussi C, Ruzzoli M, Walsh V (2010). The mechanism of transcranial magnetic stimulation in cognition. Cortex 46: 128-130.

Miniussi C, Brignani D, Pellicciari MC (2012). Combining transcranial electrical stimulation with electroencephalography: a multimodal approach. Clin EEG Neurosci 43: 184-191.

Moliadze V, Zhao Y, Eysel U et al. (2003). Effect of transcranial magnetic stimulation on single-unit activity in the cat primary visual cortex. J Physiol 553: 665-679.

Morishima Y, Akaishi R, Yamada Y et al. (2009). Taskspecific signal transmission from prefrontal cortex in visual selective attention. Nat Neurosci 12: 85-91.

Moss F, Ward LM, Sannita WG (2004). Stochastic resonance and sensory information processing: a tutorial and review of application. Clin Neurophysiol 115: 267-281.

Mottaghy FM, Gangitano M, Krause BJ et al. (2003). Chronometry of parietal and prefrontal activations in verbal working memory revealed by transcranial magnetic stimulation. Neuroimage 18: 565-575.

Nitsche MA, Paulus W (2000). Excitability changes induced in the human motor cortex by weak transcranial direct current stimulation. J Physiol 527: 633-639.

Nitsche MA, Paulus W (2011). Transcranial direct current stimulation - update 2011. Restor Neurol Neurosci 29: 463-492.

Nitsche MA, Liebetanz D, Antal A et al. (2003). Modulation of cortical excitability by weak direct current stimulation technical, safety and functional aspects. Suppl Clin Neurophysiol 56: 255-276.

Nitsche MA, Cohen LG, Wassermann EM et al. (2008). Transcranial direct current stimulation: state of the art 2008. Brain Stimul 1: 206-223.

Ohn SH, Park CI, Yoo WK et al. (2008). Time-dependent effect of transcranial direct current stimulation on the enhancement of working memory. Neuroreport 19: 43-47.

Pascual-Leone A, Bartres-Faz D, Keenan JP (1999). Transcranial magnetic stimulation: studying the brainbehaviour relationship by induction of 'virtual lesions'. Philos Trans R Soc Lond B Biol Sci 354: 1229-1238.

Pasley BN, Allen EA, Freeman RD (2009). State-dependent variability of neuronal responses to transcranial magnetic stimulation of the visual cortex. Neuron 62: 291-303.
Paulus W (2011). Transcranial electrical stimulation (tES - tDCS; tRNS, tACS) methods. Neuropsychol Rehabil 21: 602-617.

Purpura DP, McMurtry JG (1965). Intracellular activities and evoked potential changes during polarization of motor cortex. J Neurophysiol 28: 166-185.

Ridding MC, Ziemann U (2010). Determinants of the induction of cortical plasticity by non-invasive brain stimulation in healthy subjects. J Physiol 588: 2291-2304.

Romei V, Gross J, Thut G (2010). On the role of prestimulus alpha rhythms over occipito-parietal areas in visual input regulation: correlation or causation? J Neurosci 30: 8692-8697.

Rossi S, Hallett M, Rossini PM et al. (2009). Safety, ethical considerations, and application guidelines for the use of transcranial magnetic stimulation in clinical practice and research. Clin Neurophysiol 120: 2008-2039.

Rothkegel H, Sommer M, Paulus W (2010). Breaks during $5 \mathrm{~Hz}$ rTMS are essential for facilitatory after effects. Clin Neurophysiol 121: 426-430.

Rothwell JC, Day BL, Thompson PD et al. (1987). Some experiences of techniques for stimulation of the human cerebral motor cortex through the scalp. Neurosurgery 20: 156-163.

Ruohonen J (2003). Background physics for magnetic stimulation. Suppl Clin Neurophysiol 56: 3-12.

Rushmore RJ, Payne BR (2003). Bilateral impact of unilateral visual cortex lesions on the superior colliculus. Exp Brain Res 151: 542-547.

Ruzzoli M, Marzi CA, Miniussi C (2010). The neural mechanisms of the effects of transcranial magnetic stimulation on perception. J Neurophysiol 103: 2982-2989.

Ruzzoli M, Abrahamyan A, Clifford CW et al. (2011a). The effect of TMS on visual motion sensitivity: an increase in neural noise or a decrease in signal strength? J Neurophysiol 106: 138-143.

Ruzzoli M, Gori S, Pavan A et al. (2011b). The neural basis of the Enigma illusion: a transcranial magnetic stimulation study. Neuropsychologia 49: 3648-3655.

Sack AT, Linden DE (2003). Combining transcranial magnetic stimulation and functional imaging in cognitive brain research: possibilities and limitations. Brain Res Brain Res Rev 43: 41-56.

Sandrini M, Umilta C, Rusconi E (2011). The use of transcranial magnetic stimulation in cognitive neuroscience: a new synthesis of methodological issues. Neurosci Biobehav Rev 35: 516-536.

Sauseng P, Klimesch W, Stadler W et al. (2005). A shift of visual spatial attention is selectively associated with human EEG alpha activity. Eur J Neurosci 22: 2917-2926.

Schroeder CE, Lakatos P (2009). Low-frequency neuronal oscillations as instruments of sensory selection. Trends Neurosci 32: 9-18.

Schurmann M, Nikouline VV, Soljanlahti S et al. (2001). EEG responses to combined somatosensory and transcranial magnetic stimulation. Clin Neurophysiol 112: 19-24.

Schwarzkopf DS, Silvanto J, Rees G (2011). Stochastic resonance effects reveal the neural mechanisms of transcranial magnetic stimulation. J Neurosci 31: 3143-3147.

Seyal M, Masuoka LK, Browne JK (1992). Suppression of cutaneous perception by magnetic pulse stimulation of 
the human brain. Electroencephalogr Clin Neurophysiol 85: 397-401.

Sherrington CS (1910). Flexion-reflex of the limb, crossed extension-reflex, and reflex stepping and standing. J Physiol 40: 28-121.

Siebner HR, Lang N, Rizzo V et al. (2004). Preconditioning of low-frequency repetitive transcranial magnetic stimulation with transcranial direct current stimulation: evidence for homeostatic plasticity in the human motor cortex. J Neurosci 24: 3379-3385.

Siebner HR, Hartwigsen G, Kassuba T et al. (2009). How does transcranial magnetic stimulation modify neuronal activity in the brain? Implications for studies of cognition. Cortex 45: 1035-1042.

Silvanto J, Muggleton NG (2008). New light through old windows: moving beyond the "virtual lesion" approach to transcranial magnetic stimulation. Neuroimage 39: $549-552$.

Silvanto J, Muggleton NG, Cowey A et al. (2007). Neural adaptation reveals state-dependent effects of transcranial magnetic stimulation. Eur J Neurosci 25: 1874-1881.

Silvanto J, Cattaneo Z, Battelli L et al. (2008a). Baseline cortical excitability determines whether TMS disrupts or facilitates behavior. J Neurophysiol 99: 2725-2730.

Silvanto J, Muggleton N, Walsh V (2008b). State-dependency in brain stimulation studies of perception and cognition. Trends Cogn Sci 12: 447-454.

Solomon JA (2009). The history of dipper functions. Atten Percept Psychophys 71: 435-443.

Sparing R, Dafotakis M, Meister IG et al. (2008). Enhancing language performance with non-invasive brain stimulation - a transcranial direct current stimulation study in healthy humans. Neuropsychologia 46: 261-268.

Sparing R, Thimm M, Hesse MD et al. (2009). Bidirectional alterations of interhemispheric parietal balance by noninvasive cortical stimulation. Brain 132: 3011-3020.

Stein RB, Gossen ER, Jones KE (2005). Neuronal variability: noise or part of the signal? Nat Rev Neurosci 6: 389-397.

Stevens LK, McGraw PV, Ledgeway T et al. (2009). Temporal characteristics of global motion processing revealed by transcranial magnetic stimulation. Eur J Neurosci 30: 2415-2426.

Terney D, Chaieb L, Moliadze V et al. (2008). Increasing human brain excitability by transcranial high-frequency random noise stimulation. J Neurosci 28: 14147-14155.

Thickbroom GW (2007). Transcranial magnetic stimulation and synaptic plasticity: experimental framework and human models. Exp Brain Res 180: 583-593.

Thut G, Miniussi C (2009). New insights into rhythmic brain activity from TMS-EEG studies. Trends Cogn Sci 13: 182-189.

Thut G, Nietzel A, Brandt SA et al. (2006). Alpha-band electroencephalographic activity over occipital cortex indexes visuospatial attention bias and predicts visual target detection. J Neurosci 26: 9494-9502.

Thut G, Schyns PG, Gross J (2011a). Entrainment of perceptually relevant brain oscillations by non-invasive rhythmic stimulation of the human brain. Front Psychol 2: 170.
Thut G, Veniero D, Romei V et al. (2011b). Rhythmic TMS causes local entrainment of natural oscillatory signatures. Curr Biol 21: 1176-1185.

Topper R, Mottaghy FM, Brugmann M et al. (1998). Facilitation of picture naming by focal transcranial magnetic stimulation of Wernicke's area. Exp Brain Res 121: 371-378.

Varela F, Lachaux JP, Rodriguez E et al. (2001). The brainweb: phase synchronization and large-scale integration. Nat Rev Neurosci 2: 229-239.

Veniero D, Brignani D, Thut G et al. (2011). Alpha-generation as basic response-signature to transcranial magnetic stimulation (TMS) targeting the human resting motor cortex: a TMS/EEG co-registration study. Psychophysiology 48: 1381-1389.

Venkatakrishnan A, Sandrini M (2012). Combining transcranial direct current stimulation and neuroimaging: novel insights in understanding neuroplasticity. J Neurophysiol 107: 1-4.

Vines BW, Schnider NM, Schlaug G (2006). Testing for causality with transcranial direct current stimulation: pitch memory and the left supramarginal gyrus. Neuroreport 17: 1047-1050.

Walsh V, Cowey A (1998). Transcranial magnetic stimulation and cognitive neuroscience. Trends Cogn Sci 2: 103-110.

Worden MS, Foxe JJ, Wang N et al. (2000). Anticipatory biasing of visuospatial attention indexed by retinotopically specific alpha-band electroencephalography increases over occipital cortex. J Neurosci 20: RC63.

Yue L, Xiao-Lin H, Tao S (2009). The effects of chronic repetitive transcranial magnetic stimulation on glutamate and gamma-aminobutyric acid in rat brain. Brain Res [Epub ahead of print].

Zaehle T, Rach S, Herrmann CS (2010). Transcranial alternating current stimulation enhances individual alpha activity in human EEG. PLoS One 5: e13766.

Zaghi S, Acar M, Hultgren B et al. (2010). Noninvasive brain stimulation with low-intensity electrical currents: putative mechanisms of action for direct and alternating current stimulation. Neuroscientist. 16: 285-307.

Zanardini R, Gazzoli A, Ventriglia M et al. (2006). Effect of repetitive transcranial magnetic stimulation on serum brain derived neurotrophic factor in drug resistant depressed patients. J Affect Disord 91: 83-86.

Zhang X, Mei Y, Liu C et al. (2007). Effect of transcranial magnetic stimulation on the expression of c-Fos and brain-derived neurotrophic factor of the cerebral cortex in rats with cerebral infarct. J Huazhong Univ Sci Technolog Med Sci 27: 415-418.

Ziemann U, Siebner HR (2008). Modifying motor learning through gating and homeostatic metaplasticity. Brain Stimul 1: 60-66.

Ziemann U, Chen R, Cohen LG et al. (1998a). Dextromethorphan decreases the excitability of the human motor cortex. Neurology 51: 1320-1324.

Ziemann U, Hallett M, Cohen LG (1998b). Mechanisms of deafferentation-induced plasticity in human motor cortex. J Neurosci 18: 7000-7007. 